Ciência e Natura, Santa Maria, v. 37 n. 4 set-dez. 2015, p. 634-640

Revista do Centro de Ciências Naturais e Exatas - UFSM

ISSN impressa: 0100-8307 ISSN on-line: 2179-460X

\title{
ciênciaenatura
}

\section{Caracterização físico-química da cinza de casca de arroz oriunda do processo termelétrico do sul de Santa Catarina - Brasil}

\author{
Physical and chemical characterization of husk ash rice originating in the thermoelectric process in \\ south of Santa Catarina - Brazil
}

Guilherme Colle Nascimento ${ }^{1}$, Lucas Dominguini ${ }^{1}$, Josiane Maria Muneron Mello² ${ }^{2}$ Jacir Dal Magro², Humberto Gracher Riella1, Márcio Antônio Fiori²

${ }^{1}$ Post-Graduate in Chemical Engineering. Universidade Federal de Santa Catarina (UFSC) Florianópolis, 88040-900 - SC - Brazil

2 Post-Graduation in Environmental Science and Post-Graduation in Technology and Management of the Innovation. Universidade Comunitária da Região de Chapecó (UNOCHAPECO) - Chapecó, 89809-000 - SC - Brazil.

\section{Resumo}

No Sul de Santa Catarina há usinas termelétricas que empregam a casca de arroz como combustível para a geração de energia elétrica. Como resultado, milhares de toneladas de cinza de casca de arroz (CCA) são geradas como resíduo do processo termelétrico. Este artigo apresenta resultados dos estudos de caracterização da CCA com vistas na sua aplicação futura como carga de materiais poliméricos ou similares. A CCA foi doada por uma termelétrica situada no sul de Santa Catarina e foi submetida a moagem e caracterização granulométrica, a análises térmicas com calorimetria diferencial de varredura (DSC), termogravimentria (TG) $e$ dilatometria, a avaliação da estrutura por difração de raios-x e a análises química por fluorescência de raios-X. Os resultados indicaram um resíduo com baixo teor de material orgânico e com estrutura cristalina predominante contendo fases de cristobalita e quartzo. A CCA apresentou potencialidade de uso como carga após o tratamento térmico com temperaturas acima de $500{ }^{\circ} \mathrm{C}$, com a obtenção de cristobalita na fase $\beta$, ausente de material orgânico e com tamanho de partícula abaixo de $7 \mu m$.

Palavras-chave: Resíduo de termelétrica, resíduo de Casca de Arroz, cristobalita, cinza, cinza de casca de arroz, carga para compósitos.

\section{Abstract}

In the South of Santa Catarina - Brazil there are thermoelectric power that use the husk ash rice (CCA) as a fuel for electric energy generation. As a result, thousand tons of husk ash rice is generated as a residue from thermoelectric process. This article presents results of the characterizations studies for the CCA with a future objective to apply this residue as a component of composite. The CCA was donated by a thermoelectric in the South of Santa Catarina and was crushed and its size particle determined. The residue was characterized with Differential Scanning Calorimetric (DSC), Thermogravimetric Analysis (TGA) and Dilatometric Analysis. The Diffraction X-Ray Spectrometric and Fluorescence of X-Ray were applied to available the structure and chemical compositions. The results indicated the CCA being a residue containing low percentage of organic compounds and with predominance the critobalite phase. The CCA presented high potential to be applied in composite materials after its thermal treatment at temperatures above $500^{\circ} \mathrm{C}$, with cristobalyte in the phase, absent of organic materials and with particle size of $7 \mu m$.

Keywords: Thermoelectric Residues, husk rice residue, cristobalyte, husk ash rice and charge of composites. 


\section{Introdução}

A casca de arroz (CA) é um revestimento ou capa protetora formada durante o crescimento dos grãos de arroz. É composta por quatro camadas estruturais: epiderme externa, coberta com uma espessa cutícula de células silificadas; esclerênquima ou fibra hipoderme, com parede lignificada; célula parênquina esponjosa e epiderme interna ( SOUZA; MAGALHÃES; PERSEGIL, 2002). Os principais componentes da casca são celulose e hemicelulose $(50 \%)$, além de lignina $(26 \%)$ e componentes orgânicos (4\%), como óleos e proteínas. A massa restante inclui os materiais inorgânicos $\left(\mathrm{SiO}_{2}, \mathrm{Al}_{2} \mathrm{O}_{3}, \mathrm{~K}_{2} \mathrm{O}, \mathrm{Na}_{2} \mathrm{O}, \mathrm{MgO}, \mathrm{CaO}\right.$, $\mathrm{Fe}_{2} \mathrm{O}_{3}, \mathrm{MnO}_{2} \mathrm{P}_{2} \mathrm{O}_{5}$ ) (ANGEL et al., 2009).

Removidas durante o processo de beneficiamento do arroz, resultam como o subproduto mais volumoso, correspondendo a aproximadamente $23 \%$ da massa do arroz (DELLA; KÜHN; HOZTA, 2001) e contribuindo para um dos maiores problemas relacionados aos resíduos da atualidade, por ser gerado em grande quantidade pela indústria e agricultura (DELLA; KÜHN; HOZTA, 2005; UMAMAHESWARAN; BATRA, 2008). Estas cascas possuem baixo valor comercial, pois o alto percentual de sílica $\left(\mathrm{SiO}_{2}\right)$ e as fibras contidas não possuem valor nutritivo e por isso não são usadas na alimentação humana ou animal (FERRO; SILVA; WIEBECK, 2007).

Visando a utilização ou o destino adequado para esta casca, algumas formas de utilização têm sido realizadas e estudas. Este resíduo tem sido utilizado na fabricação de blocos e painéis empregados na construção civil e assim a substituição da fibra de madeira comumente utilizada (DELLA; KÜHN; HOZTA, 2001) e também para a fabricação de placas de conforto térmico para animais (ROSSI; CARDOSO; BERALDO, 2005). Porém, a forma mais utilizada, deste resíduo, é a geração de energia através da sua queima, uma alternativa praticável do ponto de vista tecnológico, uma vez que existe tecnologia para a conversão, viável do ponto de vista econômico e ecologicamente correta do ponto de vista ambiental, pois o dióxido de carbono $\left(\mathrm{CO}_{2}\right)$ produzido no processo da queima retorna para o ciclo de carbono da biosfera terrestre (FOLETTO et al., 2005). A combustão completa da casca de arroz gera gases quentes com baixos teores de contaminantes, contendo monóxido de carbono e materiais particulados (ANGEL et al., 2009).

Como produto sólido, resultante da transformação termoquímica da casca de arroz, é obtida a cinza de casca de arroz (CCA). As condições dos diferentes processos de conversão (pirólise, gaseificação e/ou combustão) determinam as características físico-químicas do produto final. A CCA corresponde de 14 a $25 \%$ da massa da casca do arroz, dependendo da variedade do arroz, do clima e do solo da região produtora (CHANDRASEKHAR et al., 2003).

Em geral, os principais elementos inorgânicos presentes nas cinzas dos combustíveis de biomassa são o $\mathrm{Ca}$, $\mathrm{K}, \mathrm{Na}$, Si e $\mathrm{P}$, sendo alguns destes importantes nutrientes para o solo (UMAMAHESWARAN; BATRA, 2008). O principal componente da CCA gerada com moderadas temperaturas de combustão é a sílica amorfa $\left(\mathrm{SiO}_{2}\right)$, em concentrações compreendidas entre 80 e 97\% (ANGEL et al., 2009). A cristalinidade e a estrutura mineral dependem das características dos processos de combustão empregados, que resulta numa estrutura mineral das CCA normalmente constituída por cristobalita, quartzo e tridimita (UMAMAHESWARAN; BATRA, 2008).

Do processo de queima podem resultar dois tipos distintos de cinza, a clara e a escura, que dependem do tempo de queima e da temperatura (RODRIGUES; BERALDO, 2010). A cinza clara é obtida com condições de temperatura de queima da ordem de 700 a 1000 ${ }^{\circ} \mathrm{C}$ e com um tempo de patamar de queima de duas horas. Esta cinza apresenta menor quantidade de carbono residual e a sílica constituinte cristaliza-se na forma de quartzo. Para temperaturas na ordem de 450 a $700{ }^{\circ} \mathrm{C}$, com um patamar de queima de 3 a 4 horas, é obtida a cinza escura, que apresenta maior quantidade de carbono residual quando comparada a cinza clara e não é cristalizada, permanecendo a sílica no estado amorfo (FERRO; SILVA; WIEBECK, 2007).

A CCA oferece diversas possiblidades de aplicação. Pode ser empregada em argamassas de revestimento como carga (KIELING et al., 2009), argamassas de colagem (RODRIGUES; BERALDO, 2010), concreto estrutural (ISAIA et al., 2010), produção de materiais refratários 
(DELLA; KÜHN; HOTZA, 2001), cargas em materiais poliméricos (FERRO; SILVA; WIEBECK, 2007; DUARTE et al., 2013) ou em borrachas naturais (ISHAK; BAKAR, 1995; ISHAK et al., 1997), produção de carbeto de silício ou até mesmo sílica pura (FOLETTO et al., 2005), produção de mulita (GONÇALVES et al., 2009) e até como adsorvente para metais pesados (CHANDRASEKHAR et al., 2003; FENG et al., 2004; KIELING; MORAES; BREHM, 2009; CHAVES et al., 2009).

Nesse contexto, o objetivo do presente trabalho é caracterizar física e quimicamente a CCA obtida durante a queima da CA em uma termoelétrica do sul de Santa Catarina, a fim de se propor uma aplicação futura deste resíduo e resguardar o meio ambiente de possíveis passivos ambientais oriundos do mal descarte desse material.

\section{Procedimento Experimental}

\subsection{Preparação da cinza de casca de arroz}

A cinza de casca de arroz (CCA) foi cedida por uma cooperativa de arroz da região sul de Santa Catarina, obtida durante o processo de geração de energia elétrica em uma usina geradora termelétrica. A cinza foi calcinada durante a queima da casca de arroz durante o próprio processo de geração que empregou a temperatura controlada de $1300{ }^{\circ} \mathrm{C}$.

Este processo permite a obtenção de cinza clara e características que indicam baixo teor de compostos orgânicos.

Após a calcinação, as amostras de cinza foram moídas em moinhos do tipo periquitos (moagem excêntrica) de capacidade de $1 \mathrm{~L}$, com carga de bolas de alta alumina fixa de $850 \pm 5 \mathrm{~g}$. O processo de moagem foi realizado com diferentes tempos para a obtenção de uma relação entre o tamanho de partícula e o tempo de moagem. A partir desta relação foi correlacionado o tamanho de partícula com o tempo de moagem e adotados os tamanhos médios de 6,9 e $12 \mu \mathrm{m}$ para os referidos estudos. Para a confirmação do tamanho de partícula da CCA foi empregada a técnica de análise de tamanho de partícula via difração de laser. $\mathrm{O}$ equipamento empregado foi um analisador de tamanho de partícula (CILAS, modelo 1064), com capacidade de medição de 500 a 0,04 $\mu \mathrm{m}$, utilizando dois feixes lasers para a realização da medida.

Após o processo de moagem, a cinza foi colocada em estufa $110 \pm 10{ }^{\circ} \mathrm{C}$ por um tempo de duas horas para garantir a não adsorção de água, sendo posteriormente empacotada, identificada e armazenada. Esta etapa foi realizada com o objetivo de avaliar a possibilidade de redução do tamanho de partícula do resíduo.

\subsection{Ensaios de caracterização da cinza de casca de arroz}

\subsubsection{Análise térmica simultânea}

Para a análise térmica simultânea as amostras de CCA foram moídas até tamanho de partículas inferiores a \#200 mesh $(75 \mu \mathrm{m})$ e secas por 1 hora em estufa em temperatura de $110 \pm 5{ }^{\circ} \mathrm{C}$. As amostras foram submetidas a ensaios térmicos em um analisador térmico simultâneo (NETZSCH, modelo STA 402 EP). As análises térmicas foram realizadas até temperaturas de $1100{ }^{\circ} \mathrm{C}$, com taxa de aquecimento de $10{ }^{\circ} \mathrm{C} / \mathrm{min}$, com atmosfera estática. Esta técnica permitiu a obtenção simultânea de curvas de análise térmica diferencial (DTA) e termogravimétrica (TGA).

\subsubsection{Dilatometria}

Para as análises de dilatometria as amostras de CCA foram prensadas com $7 \%$ de umidade e $250 \mathrm{kgf} / \mathrm{cm}^{2}$ de pressão, sendo posteriormente confeccionado o corpo de prova com dimensões de $(25 \times 5) \mathrm{mm}$. O sistema utilizado foi um dilatômetro (NETZSCH, modelo DIL 402 PC) e o ensaio realizado com taxa de aquecimento de 7,5 ${ }^{\circ} \mathrm{C} / \mathrm{min}$ em um intervalo de temperatura de 25 a $1200{ }^{\circ} \mathrm{C}$.

\subsubsection{Análise química}

A técnica empregada para a análise química da CCA foi a espectrometria de fluorescência de raios-X por dispersão de onda (FRX) em um espectrômetro (PHILIPS, modelo PW 2400), com tubos de raios $x$ de $3 \mathrm{~kW}$ e alvo de ródio com sistema de análise sequencial (com goniômetro). Para a análise, a amostra foi fundida com tetraborato de lítio, formando uma pérola, em uma proporção de 1:10 (amostra:tetraborato de 
lítio). A perda ao fogo foi realizada a $1000{ }^{\circ} \mathrm{C}$, permanecendo nesta temperatura por 1 hora.

\subsubsection{Análise mineralógica}

A amostra de CCA foi moída até tamanhos de partículas inferiores a \#200 mesh $(75 \mu \mathrm{m})$ e mantidas posteriormente em estufa, por 1 hora, em temperatura de $110 \pm 5^{\circ} \mathrm{C}$. Para a constatação das fases cristalinas no resíduo sólido estudado, foi utilizada a técnica de difração de raios-x (DRX), em difratômetro (BRUKER, modelo D8 Advance), com goniômetro theta-theta, radiação k- $\alpha$ com tubo de cobre de comprimento de onda $(\lambda)$ de $1,5406 \mathrm{~A}^{\circ}$. O passo realizado na análise foi de $0,05^{\circ}$, permanecendo em cada intervalo de ângulo por 2 segundos. O intervalo de medida foi de 2 a $72^{\circ}$, com $40 \mathrm{mV}$ e $40 \mathrm{~mA}$.

\section{Resultados e Discussão}

\subsection{Moagem}

A Tabela 1 mostra que aproximadamente $10 \%$ das partículas de CCA apresentam diâmetro médio abaixo de $2 \mu \mathrm{m}$ e que o efeito do tempo de moagem nestes valores é pequeno. Porém, um maior efeito do tempo de moagem é percebido no tamanho das partículas de CCA com valores entre 15 e $30 \mu \mathrm{m}$, que correspondem a $90 \%$.

Tabela 1: Valores dos diâmetros médios das partículas de CCA para os diferentes tempos de moagem

\begin{tabular}{cccccc}
\hline Tempo & \multicolumn{5}{c}{ Diâmetro $[\mu \mathrm{m}]$} \\
\cline { 2 - 6 }$[\mathrm{min}]$ & $10 \%$ & $50 \%$ & $90 \%$ & $100 \%$ & Médio \\
\hline 3 & 1,56 & 9,28 & 25,82 & 71,00 & 12,07 \\
5 & 1,34 & 7,05 & 20,14 & 36,00 & 9,13 \\
10 & 1,12 & 4,85 & 15,14 & 30,00 & 6,69 \\
\hline
\end{tabular}

Os resultados indicam que com 3 minutos de moagem o resíduo apresenta $90 \%$ das suas partículas com valores de tamanho médio de $25,82 \mu \mathrm{m}$ e após o tempo de 10 minutos de moagem o mesmo percentual apresenta $o$ tamanho médio de $15,14 \mu \mathrm{m}$. A redução no tamanho médio é expressiva e mostra que o tempo de moagem tem efeito no tamanho médio das partículas da CCA.

A Figura 1 mostra a tendência do tamanho de partícula média da CCA em função do tempo de moagem. Após 10 minutos de moagem o tamanho médio é de $6,69 \mu \mathrm{m}$ e a redução sendo próxima de $100 \%$ no tamanho médio das partículas do resíduo CCA.

Estes resultados comprovam a possibilidade de redução do tamanho das partículas da CCA e potencializam a sua aplicação como carga para outros materiais compósitos.

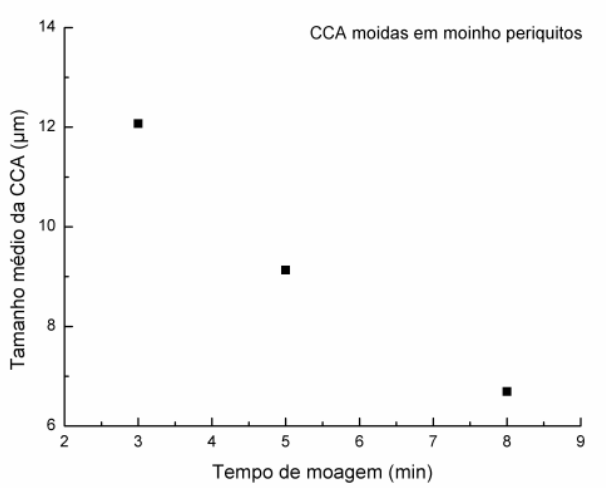

Figura 1: Relação do tamanho médio das partículas de CCA com o tempo de moagem

\subsection{Análise química}

A Tabela 2 apresenta a composição química da cinza da casca de arroz determinada por fluorescência de raios-x (FRX).

A composição química da casca de arroz varia em função das características do solo onde o arroz é plantado. Depende do tipo e teor de fertilizantes utilizados, das condições climáticas e do tipo de arroz. Os compostos de $\mathrm{K}_{2} \mathrm{O}$ e de $\mathrm{P}_{2} \mathrm{O}_{5}$ identificados nos resultados de FRX têm origem nos fertilizantes aplicados durante $\mathrm{O}$ cultivo do arroz na região (CHAVES et al., 2009).

O componente com maior teor percentual na composição da cinza da casca de arroz, após a sua calcinação no processo termelétrico, foi o dióxido de silício $\left(\mathrm{SiO}_{2}\right)$, com 86,37\%. O percentual de compostos de potássio na cinza é, da ordem de $3,09 \%$, e pode ser importante na formação de fases eutéticas da CCA a partir da cristalização das diferentes formas da sílica quando aquecidas até temperaturas de cristalização da ordem de 700 a $900{ }^{\circ} \mathrm{C}$ (ANGEL et al., 2009).

Tabela 2: Composição química da CCA determinada por fluorescência de raios $x$ 


\begin{tabular}{cc}
\hline Componente & Teor (\%) \\
\hline $\mathrm{Al}_{2} \mathrm{O}_{3}$ & 0,86 \\
$\mathrm{CaO}$ & 0,63 \\
$\mathrm{Fe}_{2} \mathrm{O}_{3}$ & 1,16 \\
$\mathrm{~K}_{2} \mathrm{O}$ & 3,09 \\
$\mathrm{MgO}$ & 0,68 \\
$\mathrm{P}_{2} \mathrm{O}_{5}$ & 4,79 \\
$\mathrm{SiO}_{2}$ & 86,37 \\
Orgânicos & 2,10 \\
\hline
\end{tabular}

$\mathrm{MnO}, \mathrm{Na}_{2} \mathrm{O}$ e $\mathrm{TiO}_{2}$ apresentaram valores inferiores a $0,15 \%$.

Os demais compostos químicos identificados na análise química também estão presentes no solo e permanecem como "contaminantes" da cinza, mesmo após o processo de queima.

\subsection{Análise mineralógica}

Os difratogramas de raios-x obtidos para a CCA demonstraram a presença de cristobalita e de quartzo, conforme mostrado na Figura 3. Um difratograma similar foi obtido e interpretado por Umamaheswaran e Batra (2008) com CCA em estudos de biomassas de origem indiana. Da mesma forma, Chaves et al. (2009) caracterizaram a CCA obtida por processo termelétrico e obtiveram como resposta uma cinza cristalizada na forma de cristobalita.

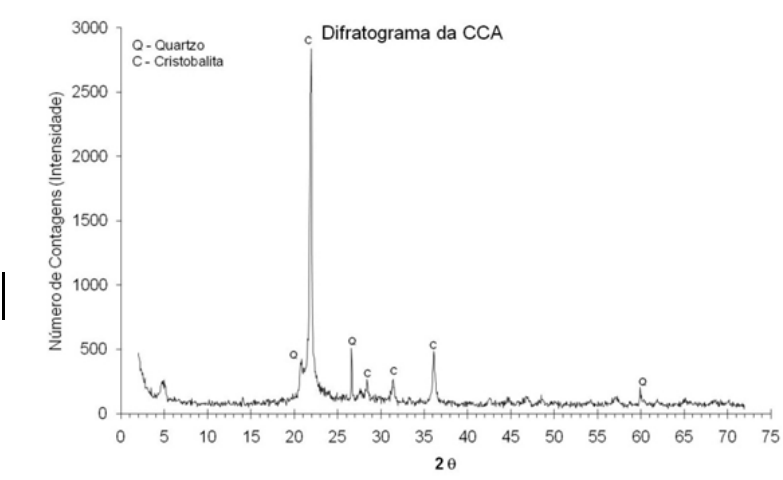

Figura 2: Difratograma de raios-x da CCA em detalhes no intervalo 2 Theta de 2 a $72^{\circ}$.

Durante o processo termelétrico a calcinação ocorreu em temperaturas elevadas da ordem de $1300{ }^{\circ} \mathrm{C}$, que associadas ao tempo de queima, favoreceram a boa cristalização, conforme demonstrado pelo difratogramas de raios-x.

Os difratogramas indicam que a CCA apresenta cristalinidade bem definida (alto grau de cristalinidade) após a sua calcinação. As fases predominantes são de cristobalita e de quartzo. Esta constatação está de acordo com os resultados encontrados por Rodrigues e Beraldo (2010).

\subsection{Análise térmica simultânea}

A Figura 3 apresenta os termogramas de DSC e de TG obtidos para a CCA com aquecimento de 25 a $1100^{\circ} \mathrm{C}$ e taxa de aquecimento de $10^{\circ} \mathrm{C} / \mathrm{min}$.

Os termogramas indicam perdas de massa da CCA durante o seu processo de aquecimento. Em, aproximadamente $130{ }^{\circ} \mathrm{C}$ o termograma indica a perda de $0,65 \%$ de massa, referente a liberação de água adsorvida na sua estrutura. Os resultados de DTA indicam um evento endotérmico em $88,1^{\circ} \mathrm{C}$, proveniente da energia absorvida pela água adsorvida e suficiente para a sua vaporização.

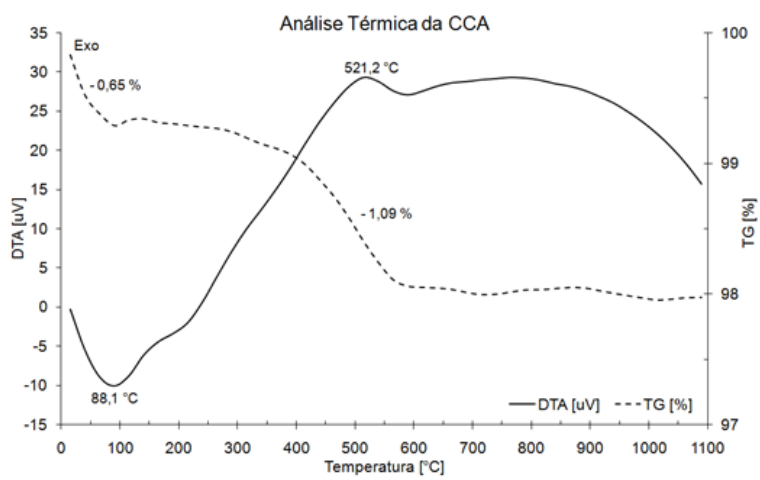

Figura 3: Termogramas de DSC e de TG obtidos para a CCA com aquecimento de 25 a $1100^{\circ} \mathrm{C} \mathrm{e}$ taxa de aquecimento de $10^{\circ} \mathrm{C} / \mathrm{min}$

No aquecimento entre a temperatura de $350{ }^{\circ} \mathrm{C}$ e $620{ }^{\circ} \mathrm{C}$ ocorre a perda de $1,09 \%$ de massa, associada com um evento exotérmico em $521,2{ }^{\circ} \mathrm{C}$. Este evento está relacionado com a volatilização dos compostos de fósforo, identificados pela análise química de FRX, e com a decomposição e volatilização dos compostos orgânicos residuais da CCA (UMAMAHESWARAN; BATRA, 2008 e DELLA; KÜHN; HOZTA, 2001).

Os termogramas das análises térmicas comprovam que o processo de queima da casca de arroz na unidade termelétrica tem boa eficiência, pois a cinza de casca de arroz, resultante da queima na termelétrica, apresenta uma quantidade muito pequena de carbono residual, o que sugere boa eficiência no ciclo de queima da casca de arroz. Verifica-se que o 
valor de perda ao fogo de 2,10\%, apresentado na análise de FRX, concorda com os resultados de perda de massa obtidos das pelas análises térmicas simultâneas, que somada as perdas de massa de $0,65 \%$ e $1,09 \%$ o valor é de $1,74 \%$.

Deve-se considerar que o pequeno evento endotérmico apresentado em $589,4^{\circ} \mathrm{C}$ está associado com a desidroxilação dos hidróxidos ainda presentes na cinza e não a queima de orgânicos.

\subsection{Dilatometria}

A curva dilatométrica, apresentada na Figura 4, ilustra uma grande expansão térmica ocorrendo no intervalo entre 180 e $240^{\circ} \mathrm{C}$.

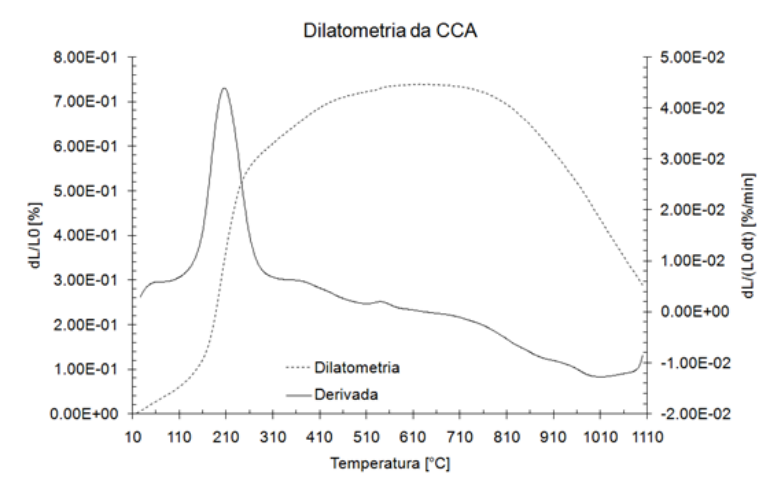

Figura 4: Curva dilatométrica da CCA, obtida em um analisador.

Esta transformação é bem definida e tem um pico de derivada bem definido em $198,4^{\circ} \mathrm{C}$. Esta transformação está associada com a mudança da fase cristobalita $\alpha$ para a fase cristobalita $\beta$. Reações de transformações entre as fases cristalinas $\alpha$ e $\beta$ correspondem às formas estáveis em baixa e alta temperatura, respectivamente. Esta transformação é rápida por ser uma distorção estrutural muito pequena dos tetraedros de $\mathrm{SiO}_{2}$ (BARBA et al., 2002). Este resultado comprova a presença da cristobalita já evidenciada pela difração de raios- $x$

Próximo a $700^{\circ} \mathrm{C}$ a estrutura da CCA deixa de expandir e inicia a retração, indicando a formação de uma fase líquida na amostra. Este comportamento é observado devido a fusão dos contaminantes da CCA, principalmente dos compostos de potássio, cálcio e fósforos. No estado óxido estes compostos são considerados fundentes e formadores de fases vítreas, uma vez que tendem a baixar o ponto de fusão (DELLA; KÜHN; HOZTA, 2001).

\section{Conclusões}

Os resultados deste trabalho indicam a possibilidade de redução do tamanho das partículas da CCA.

A partir das análises químicas por FRX e térmicas por DSC/TG é possível verificar que a massa residual de compostos orgânicos remanescentes na CCA é muito baixa, com valores da ordem de $2 \%$. Este resultado é um indicador da alta eficiência da queima da casca de arroz no processo termelétrico.

A CCA é constituída majoritariamente por sílica na fase de cristobalita. Constata-se a presença de compostos a base de potássio e fósforos oriundos dos fertilizantes empregados durante a cultura do arroz. Porém, a partir das análises térmicas verifica-se a possibilidade de volatilização destes compostos com um tratamento térmico com temperaturas superiores a $500^{\circ} \mathrm{C}$.

Assim, com o tratamento térmico da CCA é possível eliminar os compostos orgânicos residuais, os contaminantes a base de potássio e fósforo e ter como resultado um composto rico em sílica com estrutura de cristobalita $\beta$. Nestas condições, apropriado para aplicação em materiais compósitos como carga.

\section{Agradecimentos}

Os autores agradecem a equipe do Laboratório de Materiais e Corrosão (LaMAC UFSC) e o incentivo financeiro pelo Conselho Nacional de Desenvolvimento Científico e Tecnológico - CNPq.

\section{Referências}

Angel, J. D. M., Vásquez, T. G. P., Junkes, J. A., Hotza, D. (2009). Caracterização de cinza obtida por combustão de casca de arroz em reator de leito fluidizado. Química nova, 32(5), 1110-1114.

Barba, A., Beltran, V., Feliu, C., Garcia, J., Gines, F., Sánches, E., Sanz, V. (2002). Materiais primas para la fabricacion de suportes de 
baldosas cerámicas. Instituto de Tecnología Cerámica, 292.

Chandrasekhar, S., Satyanarayana, K. G., Pramada, P. N., Raghavan, P. (2003). Processing, proprieties and applications of reactive silica from rice husk - an overiew. Journal of Materials Science, 38(15), 31593168.

Chaves, T. F., Queiroz, Z. F., Sousa, D. N. R., Girão, J. H. S. (2009). Uso da cinza da casca de arroz (CCA) obtida da geração de energia térmica como adsorvente de $\mathrm{Zn}(\mathrm{II})$ em soluções aquosas. Química Nova, 32(6), 13781383.

Della, V. P., Kühn, I., Hozta, D. (2001). Caracterização de cinza de casca de arroz para uso como matéria-prima na fabricação de refratários de sílica. Química Nova, 24(6), 778-782.

Della, V. P., Kühn, I., Hozta, D. (2005). Reciclagem de resíduos agroindustriais: cinza de casca de arroz como fonte alternativa de sílica. Cerâmica Industrial, 10(2), 22-25.

Duarte, G. W., Fiori Jr., J., Peterson, M., Fiori, M. A., Colle, G. N., Riella, H. G., Neto, A. B. S. S. (2013). Effect of mass flow and mold temperature in the mechanical proprieties of polypropylene-rice rusk ash. Advances in Materials Science and Applications, 2(3), 101109.

Feng, Q., Lin, Q., Gong, F., Sugita, S., Shoya, M. (2004). Adsorption of lead and mercury by rice husk ash. Journal of Colloid and Interface Science, 278(1), 1-8.

Ferro, W. P., Silva, L. G. A., Wiebeck, H. (2007). Uso de cinza da casca de arroz como carga em matrizes de poliamida 6 e poliamida 6.6. Polímeros, 17(3), 240-243.

Foletto, E. L., Hoffmann, R., Hoffmann, R. S. Portugal Jr, U. L., Jahn, S. L. (2005). Aplicações das cinzas de casca de arroz. Química Nova, 28(6), 1055-1060.

Gonçalves, G. E., Sabioni, A. C. S., Ferraz, W. B., Costa, G. M., Brito, W., Dias, J. A., Garcia, F. A. C. (2009). Síntese e caracterização de mulita a utilizando sílica obtida da casca de arroz. Revista Escola de Minas, 62(3), 367-372.
Isaia, G. C., Gastaldini, A. L. G. Meira, L., Duart, M., Zerbino, R. (2010). Viabilidade do emprego de cinza de casca de arroz natural em concreto estrutural. Parte I: propriedades mecânicas e microestruturais. Ambiente Construído, 10(1), 121-137.

Ishak, Z. A. M., Bakar, A. A. (1995). An investigation on the potentital of rice husk ash as fillers for epoxidized natural rubber (ENR). European Polymer Journal, 31(3), 259269.

Ishak, Z. A. M., Bakar, A. A., Ishiaku, U. S., Hashim, A. S., Azahari, B. (1997). An investigation of the potential of rice husk ash as a filler for epoxidized natural rubber - II. Fatigue behavior. European Polymer Journal, 33(1), 73-79.

Kieling, A., Caetano, M. O., Kulakowski, M. P., Kasmierczak, C. S. (2009). Influência da adição de cinza da casca de arroz na aderência de argamassas de revestimento. Estudos Tecnológicos, 5(2), 157-170.

Kieling, A., Moraes, C. A. M., Brehm, F. A. (2009). Utilização de cinza de casca de arroz na remoção de cromo hexavalente. Estudos Tecnológicos, 5(3), 351-362.

Rodrigues, M. S., Beraldo, A. L. (2010). Caracterização física e mecânica de argamassas à base de cimento Portland e cinza de casca de arroz industrial. Engenharia Agrícola Jaboticabal, 30(2), 193-204.

Rossi, L. A., Cardoso, P. E. dos R., Beraldo, A. L. (2005). Engenharia Agrícola Jaboticabal, 25(1), 37-45.

Souza, M. F. de, Magalhães, W. L. E., Persegil, M. C. (2002). Silica derived from burned rice hulls. Materials Research, 5(4), 467-474.

Umamaheswaran, K., Batra, V. S. (2008). Physico-chemical characterisation of Indian biomass ashes. Fuel, 87(6), 628-638. 